\title{
Structural and magnetic characteristics of the $\mathrm{Co} / \mathrm{Cu} / \mathrm{Co}$ thin-film systems
}

\author{
Elena Shalygina ${ }^{1}$, Anna Kharlamova ${ }^{1, *}$, Andrey Makarov ${ }^{1}$, Galina Kurlyandskaya ${ }^{2}$, Andrey Svalov $^{2}$ \\ ${ }^{1}$ Faculty of Physics, Moscow State University, Moscow, Russia \\ ${ }^{2}$ Ural Federal University, Ekaterinburg, Russia
}

\begin{abstract}
The results on investigation of structural and magnetic characteristics of $\mathrm{Co} / \mathrm{Cu} / \mathrm{Co}$ thin-film systems obtained by magnetron sputtering on glass substrates are presented. The thickness of Co layers in all samples is equal to $5 \mathrm{~nm}$ and the $\mathrm{Cu}$ layer is varied from 0.5 to $4 \mathrm{~nm}$. It is found that the saturation field, $H_{\mathrm{S}}$, oscillates in magnitude with increasing $\mathrm{Cu}$ layer thickness with the period of the order of $1 \mathrm{~nm}$. The maximum values of $H_{\mathrm{S}}$ are observed for $t_{\mathrm{Cu}}=1.4,2.2$ and $3.2 \mathrm{~nm}$. The hysteresis loops measured for these samples in a magnetic field applied along the easy magnetization axis have a two-step form, and for other $t_{\mathrm{Cu}}$ - rectangular one. The obtained results are explained by the presence of exchange coupling between the ferromagnetic layers through a Co spacer and its oscillating behavior with changing $t_{\mathrm{Cu}}$.
\end{abstract}

\section{Introduction}

Magnetic thin films attracted the attention of researchers since the mid of XX century. The interest to studying these materials is due to the peculiarities of their physical properties. In particular, thin-film systems, in which the size of one of the directions is comparable with the wavelength of an electron, can exhibit quantumsize effects, which manifest themselves in a change of the energy spectrum of the samples along this direction. As a result, the transport and magnetic properties of the samples are changed. For example, giant magnetoresistance (GMR) was discovered for threelayer and multilayer thin-film systems consisting of alternating ferromagnetic (FM) and nonmagnetic (without magnetic ordering, NM) layers of submicron thicknesses. It was found that the GMR value depends on the mutual orientation of the magnetization in the FM layers and may reach several tens of percent in multilayer systems (superlattices) [1]. Furthermore, the oscillatory behavior of the exchange interaction between FM layers with a variation of NM thickness was discovered [2-4]. Due to their unique properties, multilayer thin-film systems are widely used in devices of micro- and nanoelectronics, in particular, in various sensors, including sensors of magnetic fields [5]. Thinfilm systems are also used to record and store information in the devices with high density and speed of recording [6].

A large number of experimental works are devoted to the study of the magnetic characteristics of multilayer (including a three-layer) thin-film systems. In particular, thin-film three-layer FM/NM/FM systems have been investigated in [3, 7-13], in which $\mathrm{FM}-\mathrm{Fe}$ or $\mathrm{Co}$, and $\mathrm{NM}$ - Ag, Au, Zr, Ta, Mo, Pt, Pd, Si, Bi, Cu. It was found that the values of saturation field, $H_{\mathrm{S}}$, oscillate with changing nonmagnetic layer thickness, and the period of these oscillations (except systems with $\mathrm{Bi}$ ) of the order of 1.0-1.8 nm in the thickness range of FM up to $10 \mathrm{~nm}$ and NM up to $5 \mathrm{~nm}$.

Despite the existing large volume of experimental data, the study of the magnetic properties of thin-film magnetic structures still attracts the attention of researchers from both a scientific and a practical point of view. Herewith, the most important problem is the experimental analysis of the influence of the thickness and composition of magnetic and nonmagnetic layers on the magnetic properties of thin-film magnetic systems. It is obvious that for correct solution of this question the study of two- and three-layer samples are required, since the large number of interfaces in the multilayer structures usually complicate the interpretation of the experimental data. From this point of view, three-layer samples with two magnetic cobalt layers and a copper interlayer deserved attention. The aim of this work is the investigation of influence of $\mathrm{Cu}$ interlayer on the structure and magnetic properties of the $\mathrm{Co} / \mathrm{Cu} / \mathrm{Co}$ thinfilm systems.

\section{Sample and Experimental method}

The $\mathrm{Co} / \mathrm{Cu} / \mathrm{Co}$ samples were grown by magnetron sputtering using $\mathrm{Co}$ and $\mathrm{Cu}$ targets and corning 2845 glass substrates. The Ta seed layers of $5 \mathrm{~nm}$ thick were deposited on the glass substrates. The background pressure in the vacuum chamber was $4 \cdot 10^{-7}$ mbar and the argon pressure was as high as $3.8 \cdot 10^{-3}$ mbar. A constant magnetic field of 250 Oe was applied parallel to the substrate, $H_{\mathrm{SUB}}$, in order to form an in-plane easy magnetization axis (EMA). The thickness of the Co

Kharlamova ${ }^{*}$ Corresponding author: anna-h-m@,mail.ru 
layers, $t_{\mathrm{Co}}$, in all samples was equal to $5 \mathrm{~nm}$. The thickness of the $\mathrm{Cu}$ layer, $t_{\mathrm{Cu}}$, was varied from 0.5 to 4 $\mathrm{nm}$. The microstructure of the $\mathrm{Co} / \mathrm{Cu} / \mathrm{Co}$ samples and their surface morphology were studied by X-ray diffraction (XRD) (using $\mathrm{CuK} \alpha$ radiation) and an atomic force microscope (AFM). The near-surface and the bulk magnetic characteristics of the $\mathrm{Co} / \mathrm{Cu} / \mathrm{Co}$ thin-film systems were measured employing a magneto-optical magnetometer (MOM) by means of the transverse Kerr effect and the vibration sample magnetometer «Lake Shore VSM 7400» with the sensitivity up to $10^{-7} \mathrm{G} \cdot \mathrm{cm}^{3}$. The hysteresis loops of the studied samples were measured for two directions of the magnetic field, $H$. In particular, the orientation of $H$ was parallel or perpendicular to the EMA (denoted, respectively, as D1 and D2 directions).

\section{Results and discussion}

The results of XRD measurements of $\mathrm{Co} / \mathrm{Cu} / \mathrm{Co}$ samples and «reference» monolayer Co thin film with $t_{\mathrm{Co}}=50 \mathrm{~nm}$, deposited directly on a glass substrate, are presented in Fig. 1. As can be seen in Figure 1 (b) X-ray spectra, obtained for the trilayer samples, don't have peaks corresponding to the lines of Ta. The presence of Ta seed layer enhances the texture of the Co film as the most notable remains only a peak corresponding to the line (002) of the hexagonal lattice of Co. The position of this peak for three-layer samples is slightly shifted to the left relative to the corresponding peak position for a single-layer Co film. It is evidence to the fact that at the boundary layers of $\mathrm{Ta}$ and $\mathrm{Co}$ is a partial mutual diffusion, which leads to a certain increase in the lattice parameter of Co, due to the large size of the Ta atom as compared to the Co atom. The peak on curve 3 in Fig. 1 $\mathrm{b}$, corresponding to the (111) line of the fcc lattice of $\mathrm{Cu}$, becomes noticeable. It was found that the Co films have nanocrystalline structure. The size of crystallites, estimated from the half-width of the peaks, is of the order of Co film thickness.

According to AFM investigations, the average value of the surface roughness, $R \mathrm{a}$, of the studied samples does not exceed $0.5 \mathrm{~nm}$ that also confirms high quality of the surface. The results of magnetic measurements are presented in Figure 2: MOM data in Fig. 2 a, c, e, g, i and VSM data in Fig. 2 b, d, f, h, j. All measurements were carried out in open air at room temperature. The results of magnetic measurements of hysteresis loops allowed to receive the dependences of saturation field $H_{\mathrm{S}}$ on the $t_{\mathrm{Cu}}$ (Fig. 3). As can be seen in Figure 2 the hysteresis loops, measured in the magnetic field, applied along the D1 and D2 directions, differ from each other. It should be noted that the difference of the hysteresis loops was observed at measurements by using both the magneto-optical magnetometer and the vibration one. This fact indicates the formation of induced magnetic anisotropy with EMA parallel to the direction of magnetic field, applied during the deposition process. According to the commonly accepted notions [14], the main mechanism underlying the appearance of induced magnetic anisotropy is the pair ordering of atoms.
The hysteresis loops of all the samples, measured in the magnetic field, applied along the D2 direction, have an inclined shape. Herewith, the values of coercive field, $H_{\mathrm{C}}$, and remanent magnetization are sufficiently low. In this case, the remagnetization of the samples is carried out mainly by the rotation of the magnetization vector.

The shape of hysteresis loops, measured in the magnetic field parallel to the D1 direction, depends on $t_{\mathrm{Cu}}$. In particular, some samples were characterized almost by rectangular hysteresis loops (Fig. 2 a, b, e, f), while others (Fig. 2 c, d, g, h, i, j) - by more complex two-step loops. The values of $H_{\mathrm{S}}$ of the studied samples oscillate as a function of $t_{\mathrm{Cu}}$. The peaks of $H_{\mathrm{S}}$ are observed at $t_{\mathrm{Cu}}=1.4,2.2$ and $3.2 \mathrm{~nm}$, and the hysteresis loops at these $t_{\mathrm{Cu}}$ have the two-step shape.

According to calculations, performed by K.A. Zvezdin in [15], and the experimental results [7-13, 1619] the two-step and almost rectangular hysteresis loops are observed for antiparallel and parallel orientations of magnetization components in Co layers. Thus, the obtained experimental data can be explained by the existence of exchange interaction between the FM layers through the $\mathrm{Cu}$ layer and it oscillating behavior with the change of $t_{\mathrm{Cu}}$ (transition from the ferromagnetic $(\mathrm{F})$ to the antiferromagnetic (AF) interaction).

The $\mathrm{Co} / \mathrm{Cu} / \mathrm{Co}$ samples with the ferromagnetic coupling between the magnetic layers are characterized by rectangular hysteresis loops. The remagnetization of these samples is similar to the processes of magnetization reversal of thin single- layer films, and can proceed through the irreversible growth of the remagnetization nuclei.
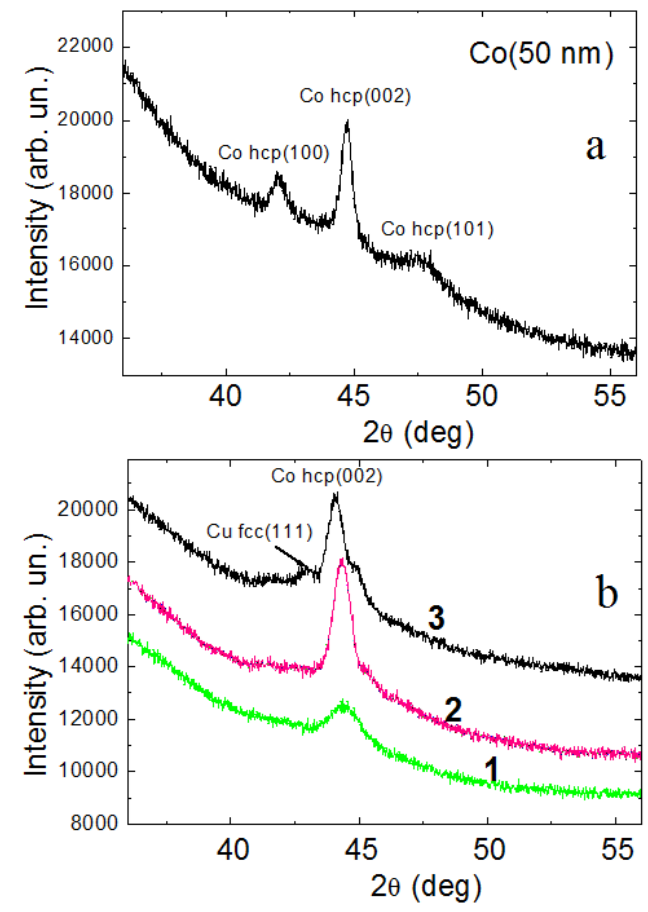

Fig. 1. Typical X-ray spectra observed for the studied samples: «reference» monolayer Co thin film with $t_{\mathrm{Co}}=50 \mathrm{~nm}$ deposited directly on a glass substrate. (a), and samples based on $\mathrm{Ta}, \mathrm{Co}$ and $\mathrm{Cu}(\mathrm{b})$ : curve $1-\mathrm{Ta} / \mathrm{Co}(5 \mathrm{~nm}) / \mathrm{Ta}$, curve $2-$ $\mathrm{Ta} / \mathrm{Co}(5 \mathrm{~nm}) / \mathrm{Cu}(1 \mathrm{~nm}) / \mathrm{Co}(5 \mathrm{~nm}), \quad$ curve 3 $\mathrm{Ta} / \mathrm{Co}(5 \mathrm{~nm}) / \mathrm{Cu}(4 \mathrm{~nm}) / \mathrm{Co}(5 \mathrm{~nm})$. 

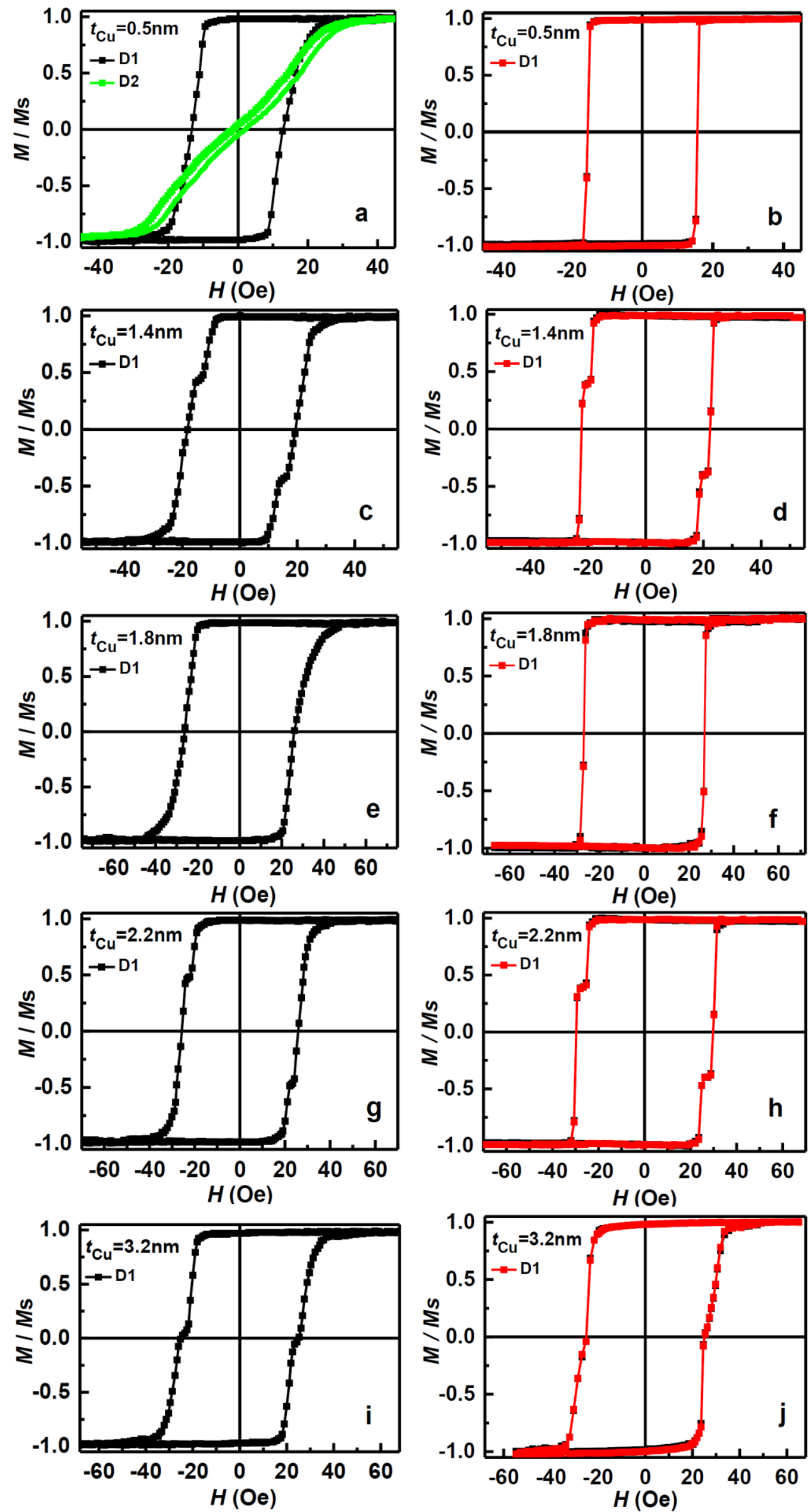

Fig. 2. Hysteresis loops of $\mathrm{Co} / \mathrm{Cu} / \mathrm{Co}$ samples measured employing magneto-optical (a, c, e, g, i) and vibration sample (b, d, f, h, j) magnetometers.

Kharlamova ${ }^{*}$ Corresponding author: anna-h-m@,mail.ru 


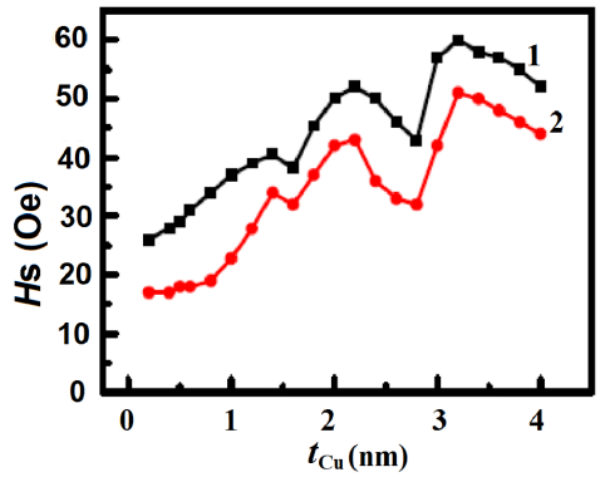

Fig. 3. Dependences of $H_{\mathrm{S}}\left(t_{\mathrm{Cu}}\right)$ obtained by using data of magneto-optical (curve 1) and vibration sample magnetometers (curve 2).

The magnetization components in Co layers, parallel to the EMA, in the samples with the antiferromagnetic coupling have the opposite directions at $H=0$, i.e., these thin-film structures are similar to uniaxial antiferromagnet. The saturation field of the samples with the antiparallel orientation of magnetization components in the Co layers (AF-exchange coupling) is higher than saturation field of the samples with the F-exchange coupling. It is caused by an additional energy, which is necessary for overcoming the AF-exchange coupling between the magnetic layers.

The analysis of experimental data presented in Figure 3 shows that the period of the oscillations of $H_{\mathrm{S}}$, $\Lambda$, is of the order of $1 \mathrm{~nm}$. It should be noted that in the multilayer systems $\mathrm{Co} / \mathrm{Pt}$ [16], $\mathrm{Fe} / \mathrm{Cr}$ [17], $\mathrm{Fe} / \mathrm{Mo}$ [18], $\mathrm{Fe} / \mathrm{Cu}[19]$ the period of the oscillations of $H_{\mathrm{S}}$ was also of the order of $1 \mathrm{~nm}$.

According to the mechanism of the exchange coupling between the magnetic layers through the nonmagnetic spacer layer via the RKKY interaction the period of the observed oscillations, $\Lambda$, must be of the order of $\pi / \kappa_{\mathrm{F}}$ [20-22], where $\kappa_{\mathrm{F}}-$ Fermi wave vector.

For most metals, $\pi / \kappa_{\mathrm{F}}$ is of the order of $0.3-0.4 \mathrm{~nm}$. The experimentally found value of $\Lambda$ is more than $\pi / \kappa_{\mathrm{F}}$. The presence of the longer period of the oscillations of $H_{\mathrm{S}}$ in the thin-film systems with metallic nonmagnetic layers was attributed to the influence of the quantum size effects, manifesting in the change of the electronic structure of ultra-thin magnetic layer (the appearance of the so-called Quantum Well States, QWSs) as compared to with bulk samples $[23,24]$. The calculated value of $\Lambda$ with taking into account the QWSs is $1-1.2 \mathrm{~nm}$.

\section{Conclusions}

The investigation of magnetic properties of the $\mathrm{Co} / \mathrm{Cu} / \mathrm{Co}$ thin-film systems have been carried out. It had been found that the values of $H_{\mathrm{S}}$ of the studied samples show oscillatory behavior with increasing $\mathrm{Cu}$ layer thickness. The dependences $H_{\mathrm{S}}\left(t_{\mathrm{Cu}}\right)$ have been explained by the exchange coupling between the ferromagnetic Co layers through the nonmagnetic $\mathrm{Cu}$ interlayer and its oscillatory behavior as a function of $t_{\mathrm{Cu}}$. The experimentally determined oscillation period of $H_{\mathrm{S}}(\Lambda \sim$ $1 \mathrm{~nm}$ ) coincided with the calculated value of $\Lambda$, obtained in theoretical works taking into account the quantum- size effects, manifesting in the change of electronic structure of ultra-thin films as compared to the bulk samples.

This work was supported by the Russian Foundation of Basic Research, Grants 15-02-02077.

\section{References}

1. S.S.P Parkin., Z.G. Li, D.J. Smith, Appl. Phys. Lett. 51, 2710 (1991).

2. S.S.P. Parkin, N. More, K.P. Roche, Phys. Rev. Lett. 64, 2304 (1990).

3. S.S.P. Parkin, R. Bhadra, K.P. Roche, Phys. Rev. Lett. 66, 2152 (1991).

4. R. Coehoorn, Ed. by K. H. J. Buschow. Elsevier, Handbook of Magnetic Materials 15, 1-197 (2003).

5. A.V. Svalov, I.R. Aseguinolaza, A. Garcia-Arribas, I. Orue et al., IEEE Trans. Magn. 46 №2, 333 (2010).

6. S.I. Kasatkin, N.P. Vasilieva, A.M. Myraviev, Institute of Control Sciences RAS 168, ISBN 585399-068-3 (2005).

7. Z.Q. Qiu, J. Pearson, S.D. Bader, Phys. Rev. B 46 № 13, 8659 (1992-I).

8. M.T. Johnson, R. Coehoorn, J.J. de Vries et al., Phys. Rev. Lett. 69 № 6, 969 (1992).

9. E.E. Shalyguina, Kyung-Ho Shin, J. Magn. Magn. Mater. 220, 167 (2000).

10. E.E. Shalygina, E.A. Gan'shina, A.M. Kharlamova et al., Solid State Phenomena 233, 653 (2015).

11. E.E Shalygina, A.V Svalov, A.M. Kharlamova et al., Japanese J. Appl. Phys. 55, 07MF01 (2016).

12. E.E. Shalygina, A.M. Kharlamova, G.V. Kurlyandskaya, A.V. Svalov, J. Magn. Magn. Mat. 440, 136 (2017).

13. W.F. Egelhoff, Jr. and M.T. Kief, IEEE Trans. Magn. 28 №5, 2742 (1992).

14. Chikazumi, Physics of Magnetism, New YorkLondon-Sydney: John Willey\&Sons, Inc. (2001)

15. A. Zvezdin. Phys. Solid State. 42, 120 (2000)

16. C.J. Lin, G.L. Gorman, C.H. Lee, R.F.C. Farrow, et al., J. Magn. Magn. Mater. 93, 194 (1991).

17. Y. Wang, P.M. Levy, J.L. Fry, Phys. Rev. Lett. 65, 2732 (1990).

18. M.E. Brubaker, J.E. Mattson, C.H. Sowers, S.D. Bader, Appl. Phys. Lett. 58, 2306 (1991).

19. W.R. Bennett, W. Schwarzacher, W.F. Egelhoff, Phys. Rev. Lett. 65, 3169 (1990).

20. M.A. Ruderman, C. Kittel, Phys. Rev. 96, 99 (1954).

21. P. Bruno, C. Chappert, Phys. Rev. Lett. 67, 1602 (1991).

22. Y. Yafet, Phys. Rev. B 36, 3948 (1987).

23. M.D. Stiles, Phys. Rev. B 48, 7238 (1993).

24. P.J. Bruno, Phys. Rev. B 52, 411 (1995).

Kharlamova ${ }^{*}$ Corresponding author: anna-h-m@,mail.ru 